\title{
A Case Study on Economic Effect of KPI for TPM Performance Measurement
}

\author{
Sangyoung $\mathrm{Oh}^{1^{*}}$, Yangkyun $\mathrm{Son}^{2}$, Yongsung $\mathrm{Suh}^{1}$ and Seonphil Jeong ${ }^{3}$ \\ ${ }^{1}$ Dept. of Business Administration, Youngdong University \\ ${ }^{2}$ Dongsung Metal Co., Ltd., ${ }^{3}$ BNU-HKBU United International College
}

\author{
사례를 통한 TPM 성과측정 $\mathrm{KPI}$ 의 경제적 효과 산출 방법 \\ 오상영 ${ }^{*}$, 손양균 ${ }^{2}$, 서용성 ${ }^{1}$, 정선필 $^{3}$ \\ ${ }^{1}$ 영동대학교 경영학과, ${ }^{2}$ (주)동성금속, ${ }^{3}$ 북경사범대-홍콩침회대 연합국제학원
}

\begin{abstract}
TPM(Total Productive Maintenance) is a methodology to improve corporations' productivity and has been employed widely. At the initial stage of the TPM, we need to set basic plans and goals of the TPM. Also, quantization of performance is required through the TPM activity measurement indices to achieve the plans and the goals. We analyzed measurement factors of TPM activity by observation of previous researches and suggest a measure methodology of economic effect for the TPM performance measurement based on BSC and quantification of qualitative measurement.
\end{abstract}

요 약 TPM 활동은 TPM의 기본 방침 및 목표의 설정과 이를 달성하기 위한 TPM 활동성과를 측정하는 지표를 통한 성과의 정량화 등이 필요하다. 본 연구에서는 TPM 활동의 측정 요소를 분석하고, BSC 기반의 TPM 성과 측정 방법과 정성적 성과의 정량화를 통한 지표의 경제적 효과 측정 방법을 제시하였다.

Key Words: TPM, BSC, Economic Effect, Quantization, KPI, Performance Measurement

\section{Introduction}

\subsection{Background}

In general, most of the companies have been making efforts on cost management for managing profit. Conventional companies, provider oriented manufacturing system, have been adopting the standard cost accounting to focus on several high weight items such as direct material cost and labour cost. However, for those companies with increasing size and complicate cost structure, they are seeking new types of cost management as consumer oriented manufacturing system. This reducing cost activity is described as 'Productivity'

Recently, Many companies have been increasing investment on informatization to improve productivity [2][13]. However, due to failure of management in investment cost and maintaining cost, numerous failing cases have been reported. [11]. Therefore, research on improving efficiency of equipment in process control has been taking place steadily such as TPM(Total Productive Maintenance). Consequently, discussion of performance measurement and economic value, which is applicable to KPI, after a whole process of TPM has drawn attention.

*Corresponding Author: Sangyoung Oh

Tel: +82-10-5486-0880 email: culture@yd.ac.kr

Received September 11, 2012 Revised October 12, 2012

Accepted November 8, 2012 


\subsection{Purpose of study}

TPM consists of 5 base principles(5S) : 'Systemizing and Standardization', 'Sorting', 'Sweeping', 'Sanitizing' and 'Self Discipline' and 8 pillars of activities : 'Autonomous Maintenance', 'Equipment and Process Improvement', 'Planned Maintenance', 'Early Management of New Equipment', 'Process Quality Management', 'Administrative and Support Departments', 'Education and Training' and 'Safety and Environmental Management' but it is very difficult to measure the performance. Moreover, several researchers had suggested evaluation tables for the performance measurement, though few methodologies exist to evaluate economic value of TPM[15].

After all, quantization of performance is required through the TPM activity measurement indices to achieve the plans and the goals.

In this study, we analyzed measurement factors of TPM activity through previous researches and suggest a measure methodology of economic effect by TPM performance measurement based on BSC and quantification of qualitative measurement.

\section{Theoretical study}

\subsection{Goals of TPM activity}

TPM(Total Productive Maintenance) has been continuously developed as a methodology of improving productivity since its introduction from Japan[6]. The ultimate goal of TPM is to increase benefit. The TPM activities such as zero breakdowns, zero disasters and zero defects during the whole facilities life cycle are able to minimize inventory costs. These systematic executions of maintenance should be done by all employees through small group activities.

\subsection{Measure factors of TPM activities}

In the previous studies, many researchers stated that quantitative performance measurement is more important than qualitative performance measurement to contribute TPM effect on business management directly[3]. However, we should not underestimate the results of qualitative performance measurement. The Quantitative indices consist of value-added productivity, fault of equipment frequency, total equipment efficiency, manufacturing defective rate, customer's complain, suspension rate, cost reduction, decreasing inventory, disaster frequency and suggestion frequency while the qualitative indices consist of more diverse factors such as mutual trust between management and labor, culture of the organization, confidence and maintenance ability to Equipment, whole employee's perception of renovation and cost, diffusion of self enrichment movement, efficient and safe operation, clearness of Equipment operation, clean process line, clear line between individual tasks and cooperation between divisions[14].

\subsection{Theories about performance measurement}

For organization performance measurement, the balance scorecard(BSC) framework has been widely used since Kaplan and Norton suggested its basic concept[9]. They have suggested four standpoints to develop metrics, collect data and analyze it relative to each of these perspectives The four perspectives are learning and growth perspective, operation process perspective, customer Perspective and financial perspective. Based on BSC, we are also able to draw Key Performance Indictors(KPI) for each of the perspectives. K. L. Sedatole found that non-financial measured values including customer satisfaction and product quality are leading indicators of finance performance[5]. While R. S. Kaplan and A. A. Atkinson insist that the performance measurement is a proper method to motivate employees and improve performances and efficiency of the organizations[9].

\subsection{BSC Perspective for TPM}

C. Parker(2000)[1] suggested systematic methodology for performance measurement in order to generate liable results. R. L. Lynch, K. F. Cross[7] emphasize the importance of effectiveness to outside and efficiency to inside of a cooperation for the performance measurement and assertion of a reasonable measurement can be accomplished with that point.

For applying BSC to performance of TPM, Manager perspective, customer perspective, performance perspective and activity perspective were selected and each perspective's success factors and measure indices are classified as follow table[14]. 
[Table 1] BSC perspective and KPI for TPM

\begin{tabular}{|c|c|c|}
\hline Perspective & $\begin{array}{l}\text { Success } \\
\text { factor }\end{array}$ & Key Performance Index (KPI) \\
\hline \multirow{2}{*}{$\begin{array}{c}\text { Managerial } \\
\text { view }\end{array}$} & profitability & $\begin{array}{l}\text { Ordinary Income, Performance } \\
\text { pay, Staff efficiency }\end{array}$ \\
\hline & Determinacy & The Sales \\
\hline \multirow{2}{*}{$\begin{array}{l}\text { Customer } \\
\text { satisfaction }\end{array}$} & Quality & Incongruent, Claim frequency \\
\hline & Time & Time for delivery rate, Lead time. \\
\hline \multirow{2}{*}{ Performance } & Manufacture & $\begin{array}{l}\text { Total Equipment efficiency, } \\
\text { Productivity, MTBF, Factory } \\
\text { disaster frequency, COD(Chemical } \\
\text { Oxygen Demand) }\end{array}$ \\
\hline & Maintenance & $\begin{array}{l}\text { Equipment examination equipment, } \\
\text { Equipment fault time, MTTR, } \\
\text { Equipment fault frequency }\end{array}$ \\
\hline \multirow{2}{*}{ Activity } & $\begin{array}{l}\text { Activity } \\
\text { efficiency }\end{array}$ & $\begin{array}{l}\text { Renovation frequency, Suggestion } \\
\text { frequency , Incongruent frequency }\end{array}$ \\
\hline & $\begin{array}{c}\text { Skill } \\
\text { Improvement }\end{array}$ & $\begin{array}{l}\text { One Point Lesson(OPL) frequency, } \\
\text { Training hours }\end{array}$ \\
\hline
\end{tabular}

\subsection{The quantification Method}

To quantify qualitative value, the Analytic Hierarchy Process(AHP) has been employed widely. This process, developed by T. L. Satty[12], is a decision-making method that observes the hierarchy structure of a decision hierarchy process based on pairwise comparison.

\section{Success factors of TPM and KPI}

\subsection{Extract measure variables}

According to Hee J. Cho's research(2007), around 500 indices are used for performance measurement widely[3] and Hee Y. Lee's case study(2009)[4] classified TPM measurement indices as performance perspective, activity perspective and skill improvement perspective. The elements of performance perspective are overall equipment efficiency(\%), labor productivity(1000 items), MTBF(time), disaster frequency(item), equipment fault frequency(item), equipment fault time(hour), MTTR(minute). The elements of activity perspective are number of renovation(item), number of suggestion(item), number of absurdity(item). In addition, the elements of skill improvement perspective are number of OPL(item) and training time per a person(hour).

\subsection{Extract factors by BSC Standard}

For this study, we surveyed TPM participants of company ' $\mathrm{D}$ ', which is a mid-sized and has vigorous TPM activity. We conducted a survey at their work places and extracted 53 cases which have more than 90\% validity among the collected data. For data analysis, we used SPSS10.1.3 and obtained cronbach $\alpha$ coefficient of 0.915 . for the questionnaire structure.

\subsection{Exploratory analysis}

We removed high correlated variables through correlation analysis. Among the five variables of managerial perspective, selling price was removed because of its strong relation with ordinary income variable. Also, among the six variables of customer perspective, customer's claim variable was selectively adopted despite of its strong correlation with incongruity rate and congruity rate. Among the nine variables of operating perspective, output variable was omitted because of its strong relation with productivity, operation rate and MTTR. Dust density was deleted resulting from its strong independence as well. Among the three strong related variables, suggestion activity rate, suggestion frequency and adoption rate of suggestion in the seven variables of measurement perspective, suggestion activity rate, and suggestion frequency were deleted and education hour variable were omitted because of its strong relation with OPL frequency.

As described above, we solved the problem of multicollinearity and performed a factor analysis. After this, four factors were sampled with eigenvalue greater than 1.0.

After the factor analysis, we named and classified measure variables with BSC standard. The factors are classified as finance, operation, customer satisfaction, education and development section. Through the literature review, we found that this classification is acceptable. In addition, we linked the factors to BSC standard for the performance measurement. 
[Table 2] Factor analysis result of the TPM activity's importance

\begin{tabular}{|c|c|c|c|c|c|c|}
\hline \multirow{2}{*}{ Factors } & \multirow{2}{*}{$\begin{array}{l}\text { Measure } \\
\text { Variables }\end{array}$} & \multicolumn{4}{|c|}{ Composition } & \multirow{2}{*}{$\begin{array}{c}\text { Common } \\
\text { ness }\end{array}$} \\
\hline & & Fac.1 & Fac. 2 & Fac.3 & Fac.4 & \\
\hline \multirow{4}{*}{$\begin{array}{c}\text { Financial } \\
\text { Factors }\end{array}$} & $\begin{array}{l}\text { O r d i in a r y } \\
\text { Income }(1)^{1)}\end{array}$ & 0.880 & 0.056 & 0.083 & 0.220 & 0.833 \\
\hline & \begin{tabular}{|l|} 
Manufacturing \\
Cost(2)
\end{tabular} & 0.811 & 0.138 & 0.308 & 0.093 & 0.781 \\
\hline & \begin{tabular}{|l|}
$\begin{array}{l}\text { Cost reduction } \\
(10)\end{array}$ \\
\end{tabular} & 0.749 & 0.158 & 0.336 & 0.100 & 0.709 \\
\hline & $\begin{array}{|ll|}\text { HR } & \text { Efficiency } \\
(15) & \\
\end{array}$ & 0.507 & 0.108 & 0.295 & 0.496 & 0.602 \\
\hline \multirow{6}{*}{$\begin{array}{l}\text { Operation } \\
\text { Process } \\
\text { Factors }\end{array}$} & $\begin{array}{l}\text { Equ i p m e n t } \\
\text { Efficiency (3) }\end{array}$ & 0.069 & 0.816 & 0.314 & 0.145 & 0.790 \\
\hline & MTBF(8) & -0.037 & 0.780 & 0.120 & 0.131 & 0.642 \\
\hline & $\begin{array}{l}\text { Equ i p m e n t } \\
\text { Productivity } \\
\text { (9) }\end{array}$ & 0.090 & 0.686 & 0.402 & 0.039 & 0.642 \\
\hline & $\begin{array}{l}\text { Number } \\
\text { Disaster(11) }\end{array}$ & 0.491 & 0.634 & -0.073 & 0.234 & 0.702 \\
\hline & \begin{tabular}{|l|}
$\begin{array}{l}\text { Disaster Ration } \\
\text { (13) }\end{array}$ \\
\end{tabular} & 0.455 & 0.631 & -0.182 & 0.151 & 0.661 \\
\hline & MTTR(17) & 0.263 & 0.551 & 0.224 & 0.355 & 0.548 \\
\hline \multirow{4}{*}{$\begin{array}{l}\text { Customers } \\
\text { Satisfactio } \\
\text { n Factors }\end{array}$} & \begin{tabular}{|lr}
$\begin{array}{l}\text { Number } \\
\text { Claim (4) }\end{array}$ & of \\
\end{tabular} & 0.058 & 0.216 & 0.829 & 0.099 & 0.747 \\
\hline & \begin{tabular}{|l|} 
Cus t o r m e r \\
Stisfaction (7) \\
\end{tabular} & 0.193 & 0.169 & 0.798 & 0.050 & 0.705 \\
\hline & \begin{tabular}{|l|} 
Time delivery \\
Ratio (14)
\end{tabular} & 0.226 & 0.223 & 0.696 & 0.308 & 0.680 \\
\hline & $\begin{array}{l}\begin{array}{l}\text { In v e n t r o y } \\
\text { period(16) }\end{array} \\
\end{array}$ & 0.276 & -0.116 & 0.599 & 0.431 & 0.635 \\
\hline \multirow{4}{*}{$\begin{array}{l}\text { Training } \\
\text { and } \\
\text { Growth } \\
\text { Factors }\end{array}$} & $\begin{array}{l}\text { Number of } \\
\text { renonovation } \\
(5)\end{array}$ & 0.064 & 0.219 & 0.111 & 0.824 & 0.743 \\
\hline & $\begin{array}{l}\text { Suggestion } \\
\text { A d o p t i o n } \\
\text { Ration (6) }\end{array}$ & 0.539 & 0.155 & 0.186 & 0.568 & 0.672 \\
\hline & $\begin{array}{l}\text { Number of } \\
\text { Irrationality } \\
\mathrm{y}(12) \\
\end{array}$ & 0.171 & 0.202 & 0.360 & 0.561 & 0.515 \\
\hline & $\begin{array}{|ll|}\text { Number } & \text { of } \\
\text { OPL (18) }\end{array}$ & 0.510 & 0.275 & -0.017 & 0.544 & 0.632 \\
\hline \multicolumn{2}{|c|}{ Eigen value } & 3.534 & 3.233 & 3.010 & 2460 & \\
\hline
\end{tabular}

* ${ }^{1)}$ Number of Questionnaire

\subsection{Definition of KPI}

\subsubsection{KPI of TPM performance measurement}

Selection of KPI should be done in accordance with the organizations' goals and focused on core activities of the organizations. Also, relationship among the core success factors should be considered and every single activity should be examined. Validity of performance measurement should be proved and control possibility of performance indicators, which are distributed as top-down model should be provided.

To make the performance measurement clear by KPI indicators, there are six items for consideration. 1) Proper number of KPI should be suggested 2) strategy and CSF should be linked 3) change and flow of past, present and future should be reflected 4) The indices should be amendable. 5) It should be able to motivate the organization. 6) It should be able to classify responsibility effectively[15].

\section{Case Study}

\subsection{Selection KPI for company ' $D$ '}

H.Y Lee research(2009), a study focus on TPM of Equipment-dependant cooperation, applied for analyzing the first term of $\operatorname{TPM}(2001 \sim 2008)$ of the company 'D', which is also very equipment dependant. The analysis proved that the first term of TPM activity was the core of the management renovation. We surveyed 'D's managers about the importance of KPI and we were able to extract the KPI for the performance measurement of the 'D'.

\subsubsection{KPI Analysis}

As table 3, we used $18 \mathrm{KPI}$, as questionnaires about importance and data collection. We interviewed 17 managers of the 'D' from May 25th to May 31st. At the first time, we had a group interview with all the participants to explain the purpose of this survey. Also, the managers were given time to review reported problem during the last term of the TPM activity. Consequently, we had individual interview and omitted disaster frequency, man power efficiency and OPL frequency due to their relatively lower mean value and lower standard deviation(SD).

[Table 3] Result of factor importance analysis for KPI selection

\begin{tabular}{|c|l|c|c|c|c|c|}
\hline No & \multicolumn{1}{|c|}{ Factors } & Ma & SD & $\begin{array}{c}\text { Ma } \\
\text { rank }\end{array}$ & $\begin{array}{c}\text { SD } \\
\text { rank }\end{array}$ & $\begin{array}{c}\text { Accept } \\
\text { ance }\end{array}$ \\
\hline 1 & Ordinary income & 3.98 & 0.951 & 11 & 15 & Acc. \\
\hline 2 & Manufacturing cost & 4.19 & 0.942 & 2 & 14 & Acc. \\
\hline 3 & Equipment efficiency & 4.06 & 0.842 & 7 & 5 & Acc. \\
\hline 4 & Claim frequency & 4.02 & 0.888 & 9 & 7 & Acc. \\
\hline 5 & Renovation Frequency & 4.26 & 0.738 & 1 & 1 & Acc. \\
\hline 6 & $\begin{array}{l}\text { Suggestion adoption } \\
\text { rate }\end{array}$ & 4.11 & 0.800 & 5 & 2 & Acc. \\
\hline
\end{tabular}




\begin{tabular}{|c|l|c|c|c|c|c|}
\hline 7 & $\begin{array}{l}\text { Customer satisfaction } \\
\text { rate }\end{array}$ & 3.87 & 0.941 & 13 & 13 & Acc. \\
\hline 8 & MTBF & 4.13 & 0.810 & 4 & 4 & Acc. \\
\hline 9 & Productivity & 4.00 & 0.899 & 10 & 8 & Acc. \\
\hline 10 & Amount cost reduction & 4.06 & 0.908 & 6 & 11 & Acc. \\
\hline 11 & Disaster frequency & 3.70 & 0.972 & 18 & 16 & Rej. \\
\hline 12 & Number of Irrationality & 4.13 & 0.810 & 3 & 3 & Acc. \\
\hline 13 & Disaster rate & 3.91 & 0.925 & 12 & 12 & Acc. \\
\hline 14 & Timely delivery rate & 3.81 & 0.878 & 15 & 6 & Acc. \\
\hline 15 & HR efficiency & 3.75 & 0.998 & 17 & 18 & Rej. \\
\hline 16 & Inventory period & 4.06 & 0.908 & 8 & 10 & Acc. \\
\hline 17 & MTTR & 3.87 & 0.900 & 14 & 9 & Acc. \\
\hline 18 & OPL frequency & 3.77 & 0.974 & 16 & 17 & Rej. \\
\hline
\end{tabular}

${ }^{*}$ Low factors are highlighted.

Based on the above results, we selected analysis factors for the performance measurement and KPI for the company 'D'. The KPI perspectives and descriptions are shown in the below table.

[Table 4] Formulas for KPI to measure TPM performance of company' D'

\begin{tabular}{|c|c|c|}
\hline Perspective & KPI & Formula \\
\hline \multirow{3}{*}{$\begin{array}{c}\text { Financial } \\
\text { view }\end{array}$} & $\begin{array}{l}\text { Ordinary } \\
\text { income }\end{array}$ & $\begin{array}{l}\text { (This year ordinary income - Last year } \\
\text { ordinary income)/Last year ordinary } \\
\text { income *100 }\end{array}$ \\
\hline & $\begin{array}{c}\text { Manufacturi } \\
\text { ng } \\
\text { Cost rate }\end{array}$ & $\begin{array}{l}\text { (This year manufacturing cost - Last } \\
\text { year Manufacturing cost)/Last year } \\
\text { Manufacturing cost* } 100\end{array}$ \\
\hline & $\begin{array}{l}\text { Amount of } \\
\text { Cost } \\
\text { Reduction }\end{array}$ & $\begin{array}{l}\text { (This year manufacturing cost - Last } \\
\text { year amount of cost reduction)/Last } \\
\text { year amount of cost reduction* } 100\end{array}$ \\
\hline \multirow{5}{*}{ Operation } & $\begin{array}{l}\text { Overall } \\
\text { equipment } \\
\text { efficiency }\end{array}$ & $\begin{array}{l}\text { Equipment operation rate } \times \text { Equipment } \\
\text { Performacne rate } \times \text { non-defective parts } \\
\text { or products ratio }\end{array}$ \\
\hline & MTBF & $\begin{array}{l}\text { (Equipment uptime - Time to } \\
\text { failure)/Number of equipment fault }\end{array}$ \\
\hline & $\begin{array}{c}\text { Equipment } \\
\text { productivity }\end{array}$ & $\begin{array}{l}\text { (This year equipment productivity- Last } \\
\text { year equipment productivity)/This year } \\
\text { equipment productivity } * 100\end{array}$ \\
\hline & $\begin{array}{l}\text { Disaster } \\
\text { Frequency }\end{array}$ & $\begin{array}{l}\text { (Number of this year disaster-Number } \\
\text { of last year disaster)/Number of last } \\
\text { year disaster*100 }\end{array}$ \\
\hline & MTTR & Total suspended time/suspended item \\
\hline
\end{tabular}

\begin{tabular}{|c|c|c|}
\hline \multirow{4}{*}{$\begin{array}{c}\text { Customer } \\
\text { satisfaction }\end{array}$} & $\begin{array}{c}\text { Claim } \\
\text { Frequency }\end{array}$ & Number of Claim \\
\hline & $\begin{array}{l}\text { Customer } \\
\text { Satisfaction } \\
\text { Rate }\end{array}$ & $\begin{array}{l}\text { Customers feedback } \\
\text { (Qualitative measure) }\end{array}$ \\
\hline & $\begin{array}{l}\text { Timely } \\
\text { delivery } \\
\text { rate }\end{array}$ & Delayed delivery dates \\
\hline & $\begin{array}{l}\text { Inventory } \\
\text { period }\end{array}$ & Inventory period after prodcution \\
\hline \multirow{3}{*}{$\begin{array}{c}\text { Learning } \\
\text { and Growth }\end{array}$} & $\begin{array}{r}\text { Renovation } \\
\text { Frequency }\end{array}$ & $\begin{array}{l}\text { (Number of this year renovation-Number } \\
\text { of this year renovation)/Number of this } \\
\text { year renovation *100 }\end{array}$ \\
\hline & $\begin{array}{l}\text { Suggestion } \\
\text { Adoption } \\
\text { rate }\end{array}$ & $\begin{array}{l}\text { Number of Yearly Adoption / Number } \\
\text { of Whole period Adoption * } 100\end{array}$ \\
\hline & $\begin{array}{r}\text { Irrationality } \\
\text { Frequency }\end{array}$ & $\begin{array}{l}\text { Number of Yearly Irrationality / } \\
\text { Number of Whole Irrationality * } 100\end{array}$ \\
\hline
\end{tabular}

\subsection{Performance decision by economic value analysis}

Ordinary income and reduction cost are economic variables and the rest of the variables are quantitative or qualitative variables among the above 15 measurement variables.

We used AHP method to draw weight of KPI by the perspectives. Two responses, with higher consistency rate than 1.0, among the 12 respondents were deleted. With the 10 responses, we drew weight of the variables first as shown in the table 5. Also, we selected 5 respondents who have longest work experience to decide the weight of KPI again. All the Consistency rates of the selected weights are less than 0.1 .

[Table 5] The Weight of the KPI by perspectives

\begin{tabular}{|l|c|l|c|c|}
\hline Perspective & $\begin{array}{c}\text { Weight } \\
\mathbf{( \% )}\end{array}$ & \multicolumn{1}{|c|}{ KPI } & $\begin{array}{c}\text { Weight } \\
\mathbf{( \% )}\end{array}$ & $\begin{array}{c}\text { Total } \\
\text { weight } \\
(\mathbf{\%})\end{array}$ \\
\hline \multirow{3}{*}{$\begin{array}{c}\text { Financial } \\
\text { view }\end{array}$} & \multirow{2}{*}{25.8} & Ordinary income & 0.58 & 14.9 \\
\cline { 3 - 5 } & & manufacturing cost rate & 0.12 & 3.2 \\
\cline { 3 - 5 } & Amount cost reduction & 0.30 & 7.7 \\
\hline \multirow{3}{*}{$\begin{array}{c}\text { Operation } \\
\text { Process }\end{array}$} & \multirow{3}{*}{43.5} & Equipment efficiency & 0.10 & 4.5 \\
\cline { 3 - 5 } & MTBF & 0.26 & 11.3 \\
\cline { 3 - 5 } & Equipment productivity & 0.34 & 14.6 \\
\cline { 3 - 5 } & $\begin{array}{l}\text { Disaster } \\
\text { Frequency }\end{array}$ & 0.12 & 5.0 \\
\cline { 3 - 5 } & MTTR & 0.19 & 8.0 \\
\hline
\end{tabular}


A Case Study on Economic Effect of KPI for TPM Performance Measurement

\begin{tabular}{|l|l|l|c|c|}
\hline \multirow{4}{*}{$\begin{array}{c}\text { Customer } \\
\text { satisfaction }\end{array}$} & \multirow{3}{*}{19.9} & Number of Claim & 0.24 & 4.7 \\
\cline { 3 - 5 } & & Customer satisfaction & 0.42 & 8.3 \\
\cline { 3 - 5 } & & Timely delivery rate & 0.24 & 4.9 \\
\cline { 3 - 5 } $\begin{array}{c}\text { Learning } \\
\text { and Growth }\end{array}$ & \multirow{2}{*}{10.8} & Inventory period & 0.10 & 2.0 \\
\cline { 3 - 5 } & & Number of renovation & 0.35 & 3.8 \\
\cline { 3 - 5 } & Suggestion adoption rate & 0.36 & 3.9 \\
\cline { 3 - 5 } & Number of irrationality & 0.29 & 3.1 \\
\hline
\end{tabular}

To measure economic value of performance measurement factors, we calculated all the contribution degree of variables through the conducted interview with the 12 respondents. The results are shown in the table 6 which were rounded up.

[Table 6] Contribution weight of measure variables

\begin{tabular}{|c|c|c|c|c|c|c|c|c|c|c|c|c|c|c|c|}
\hline \multirow[t]{2}{*}{$\mathrm{n}$} & \multicolumn{3}{|c|}{$\begin{array}{c}\text { Financial } \\
\text { view }\end{array}$} & \multicolumn{5}{|c|}{$\begin{array}{c}\text { Operation } \\
\text { process }\end{array}$} & \multicolumn{4}{|c|}{$\begin{array}{c}\text { Customer } \\
\text { satisfaction }\end{array}$} & \multicolumn{3}{|c|}{$\begin{array}{c}\text { Learning and } \\
\text { Growth }\end{array}$} \\
\hline & $\mathrm{A}$ & $\mathrm{B}$ & $\mathrm{C}$ & $\mathrm{D}$ & $\mathrm{E}$ & $\mathrm{F}$ & $\mathrm{G}$ & $\mathrm{H}$ & $\mathrm{I}$ & $\mathrm{J}$ & $\mathrm{K}$ & $\mathrm{L}$ & $\mathrm{M}$ & $\mathrm{N}$ & 0 \\
\hline 1 & 5 & 4 & 6 & 5 & 4 & 8 & 3 & 3 & 4 & 4 & 2 & 2 & 4 & 4 & 2 \\
\hline 2 & 4 & 3 & 6 & 5 & 3 & 7 & 3 & 3 & 5 & 5 & 2 & 2 & 4 & 3 & 3 \\
\hline 3 & 3 & 3 & 6 & 4 & 3 & 7 & 3 & 3 & 3 & 5 & 2 & 2 & 3 & 4 & 2 \\
\hline 4 & 5 & 4 & 6 & 5 & 5 & 7 & 3 & 3 & 4 & 5 & 2 & 2 & 4 & 4 & 2 \\
\hline 5 & 4 & 4 & 5 & 4 & 4 & 6 & 4 & 2 & 3 & 4 & 3 & 1 & 3 & 3 & 1 \\
\hline 6 & 5 & 4 & 4 & 6 & 3 & 5 & 2 & 3 & 4 & 5 & 1 & 3 & 4 & 4 & 3 \\
\hline 7 & 4 & 2 & 5 & 4 & 3 & 6 & 2 & 2 & 3 & 4 & 1 & 1 & 3 & 3 & 1 \\
\hline 8 & 5 & 3 & 7 & 5 & 4 & 6 & 2 & 2 & 3 & 4 & 1 & 1 & 3 & 3 & 1 \\
\hline 9 & 4 & 1 & 4 & 5 & 2 & 5 & 1 & 1 & 3 & 5 & 1 & 3 & 3 & 4 & 2 \\
\hline 10 & 4 & 3 & 6 & 5 & 4 & 7 & 3 & 3 & 4 & 4 & 2 & 2 & 4 & 4 & 2 \\
\hline 11 & 4 & 3 & 6 & 4 & 4 & 7 & 3 & 3 & 3 & 5 & 2 & 2 & 4 & 4 & 3 \\
\hline 12 & 7 & 3 & 6 & 4 & 4 & 6 & 3 & 3 & 4 & 5 & 3 & 2 & 4 & 4 & 2 \\
\hline Averag & 5 & 3 & 6 & 5 & 4 & 6 & 3 & 3 & 4 & 5 & 2 & 2 & 4 & 4 & 2 \\
\hline
\end{tabular}

* A:Ordinary income, B:Manufacturing Cost rate , C::Amount of Cost Reduction, D:Overall equipment efficiency, E::MTBF, F:Equipment productivity, G: Disaster Frequency, H: MTTR, I: Number of Claim, J: Customer satisfaction, K: Timely delivery rate, L: Inventory period, M: Number of renovation, $\mathrm{N}$ : Suggestion adoption rate, O: Number of irrationality

Then, we estimated economic value by comparing analysis of quantification ratio based on contribution and weight of analyzed qualitative and qualitative effect.

However, ordinary income is the only economic factor. Consequently, we recalculated the weight including the ordinary income factor to find out the economic value by each perspective.

Finally, table 7 shows expectation of economic effect based on the four perspectives.
[Table 7] Economic effect of financial perspective

\begin{tabular}{|c|c|c|c|c|c|}
\hline \multirow[b]{2}{*}{ Classification } & \multirow{2}{*}{$\begin{array}{l}\text { Weight of } \\
\text { index } \\
\text { (a) }\end{array}$} & \multirow{2}{*}{$\begin{array}{l}\text { Contri } \\
\text { bution } \\
\text { (b) }\end{array}$} & \multirow[b]{2}{*}{$(\mathrm{a}) *(\mathrm{~b})$} & \multicolumn{2}{|c|}{$\begin{array}{c}\text { Quantization of } \\
\text { Qualitative variables } \\
\end{array}$} \\
\hline & & & & \begin{tabular}{|l|} 
Quantitat \\
ive rate
\end{tabular} & $\begin{array}{l}\text { economic } \\
\text { value(Mill } \\
\text { on Won) }\end{array}$ \\
\hline $\begin{array}{l}\text { Economic } \\
\text { Index }\end{array}$ & $\begin{array}{c}\text { Ordinary } \\
\text { income } \\
(62.0 \%) \\
\end{array}$ & 5 & 3.10 & 1.00 & 1,200 \\
\hline $\begin{array}{l}\text { Quantitative } \\
\text { Index }\end{array}$ & \begin{tabular}{|c} 
Manufacturi \\
ng Cost \\
$(15.6 \%)$ \\
\end{tabular} & 3 & 0.47 & 0.15 & 181 \\
\hline $\begin{array}{l}\text { Quantitative } \\
\text { index }\end{array}$ & $\begin{array}{c}\text { Amount of } \\
\text { cost } \\
\text { reduction } \\
(22.4 \%)\end{array}$ & 6 & 1.35 & 0.43 & 521 \\
\hline \multicolumn{5}{|c|}{ Total } & 1,902 \\
\hline
\end{tabular}

[Table 8] Economic effect of operation process perspective

\begin{tabular}{|c|c|c|c|c|c|}
\hline \multirow{2}{*}{ Classification } & \multirow{2}{*}{$\begin{array}{l}\text { Weight of index } \\
\text { (a) }\end{array}$} & \multirow{2}{*}{$\begin{array}{l}\text { Contri } \\
\text { bution } \\
\text { (b) }\end{array}$} & \multirow{2}{*}{$(a)^{*}(b)$} & \multicolumn{2}{|c|}{$\begin{array}{l}\text { Quantization of } \\
\text { Qualitative } \\
\text { variables }\end{array}$} \\
\hline & & & & $\begin{array}{l}\text { Quant } \\
\text { itative } \\
\text { rate }\end{array}$ & $\begin{array}{l}\text { economic } \\
\text { value(Mil } \\
\text { lon Won) }\end{array}$ \\
\hline $\begin{array}{l}\text { Economic } \\
\text { Index }\end{array}$ & $\begin{array}{c}\text { Ordinary income } \\
(28.7 \%)\end{array}$ & 5 & 1.44 & 1.00 & 1,200 \\
\hline $\begin{array}{c}\text { Quantitative } \\
\text { Index }\end{array}$ & $\begin{array}{c}\text { Overall } \\
\text { equipment } \\
\text { efficiency }(5.4 \%)\end{array}$ & 5 & 0.27 & 0.19 & 225 \\
\hline $\begin{array}{c}\text { Quantitative } \\
\text { Index }\end{array}$ & $\begin{array}{l}\text { MTBF } \\
(18.4 \%)\end{array}$ & 4 & 0.73 & 0.51 & 614 \\
\hline $\begin{array}{l}\text { Quantitative } \\
\text { Index }\end{array}$ & $\begin{array}{l}\text { Equipment } \\
\text { productivity } \\
(32.4 \%)\end{array}$ & 6 & 1.94 & 1.35 & 1,625 \\
\hline $\begin{array}{l}\text { Quantitative } \\
\text { Index }\end{array}$ & $\begin{array}{c}\text { Disaster } \\
\text { Frequency } \\
(6.4 \%)\end{array}$ & 3 & 0.19 & 0.13 & 160 \\
\hline $\begin{array}{c}\text { Quantitative } \\
\text { Index }\end{array}$ & $\begin{array}{l}\text { MTTR } \\
(8.8 \%)\end{array}$ & 3 & 0.26 & 0.18 & 220 \\
\hline \multicolumn{5}{|c|}{ Total } & 4,044 \\
\hline
\end{tabular}

[Table 9] Economic effect of customer satisfaction perspective

\begin{tabular}{|c|c|c|c|c|c|}
\hline \multirow[b]{2}{*}{ Classification } & \multirow{2}{*}{$\begin{array}{l}\text { Weight of } \\
\text { index } \\
\text { (a) }\end{array}$} & \multirow{2}{*}{$\begin{array}{c}\text { Contri } \\
\text { bution } \\
\text { (b) }\end{array}$} & \multirow[b]{2}{*}{$(a)^{*}(b)$} & \multicolumn{2}{|c|}{$\begin{array}{c}\text { Quantization of } \\
\text { Qualitative variables }\end{array}$} \\
\hline & & & & $\begin{array}{l}\text { Quanti } \\
\text { zation } \\
\text { rate }\end{array}$ & $\begin{array}{c}\text { Economic } \\
\text { value } \\
\text { (Million Won) }\end{array}$ \\
\hline $\begin{array}{l}\text { Economic } \\
\text { Index }\end{array}$ & $\begin{array}{l}\text { Ordinary } \\
\text { income } \\
(31.4 \%)\end{array}$ & 5 & 1.57 & 1.00 & 1,200 \\
\hline $\begin{array}{l}\text { Quantitative } \\
\text { Index }\end{array}$ & $\begin{array}{c}\text { Number of } \\
\text { Claim } \\
(11.7 \%)\end{array}$ & 4 & 0.47 & 0.30 & 358 \\
\hline $\begin{array}{l}\text { Qualitative } \\
\text { Index }\end{array}$ & \begin{tabular}{|c|} 
Customer \\
satisfaction \\
$(23.6 \%)$ \\
\end{tabular} & 5 & 1.18 & 0.75 & 902 \\
\hline $\begin{array}{l}\text { Quantitative } \\
\text { Index }\end{array}$ & $\begin{array}{c}\text { Timely } \\
\text { delivery } \\
\text { rate } \\
(17.5 \%)\end{array}$ & 2 & 0.35 & 0.22 & 267 \\
\hline $\begin{array}{l}\text { Quantitative } \\
\text { Index }\end{array}$ & \begin{tabular}{|c|} 
Inventory \\
period \\
$(15.8 \%)$ \\
\end{tabular} & 2 & 0.32 & 0.20 & 242 \\
\hline \multicolumn{5}{|c|}{ Total } & 2,699 \\
\hline
\end{tabular}


[Table 10] Economic effect of learning and growth perspective

\begin{tabular}{|c|c|c|c|c|c|}
\hline \multirow[b]{2}{*}{ Classification } & \multirow{2}{*}{$\begin{array}{l}\text { Weight of } \\
\text { index } \\
\text { (a) }\end{array}$} & \multirow{2}{*}{$\begin{array}{l}\text { Contri } \\
\text { bution } \\
\text { (b) }\end{array}$} & \multirow[b]{2}{*}{$(a) *(b)$} & \multicolumn{2}{|c|}{$\begin{array}{c}\text { Quantization of } \\
\text { Qualitative } \\
\text { variables }\end{array}$} \\
\hline & & & & $\begin{array}{l}\text { Quantit } \\
\text { ative } \\
\text { rate }\end{array}$ & $\begin{array}{l}\text { economic } \\
\text { value } \\
\text { (Millon } \\
\text { Won) }\end{array}$ \\
\hline $\begin{array}{l}\text { Economic } \\
\text { Index }\end{array}$ & $\begin{array}{l}\text { Ordinary } \\
\text { income } \\
(66.9 \%)\end{array}$ & 5 & 3.35 & 1.00 & 1,200 \\
\hline $\begin{array}{l}\text { Quantitative } \\
\text { Index }\end{array}$ & $\begin{array}{l}\text { Number of } \\
\text { renovation } \\
(13.9 \%)\end{array}$ & 4 & 0.56 & 0.17 & 1.99 \\
\hline $\begin{array}{l}\text { Quantitative } \\
\text { Index }\end{array}$ & $\begin{array}{c}\text { Suggestion } \\
\text { adoption rate } \\
(9.8 \%)\end{array}$ & 4 & 0.39 & 0.12 & 1.41 \\
\hline $\begin{array}{l}\text { Quantitative } \\
\text { Index }\end{array}$ & $\begin{array}{l}\text { Number of } \\
\text { irrationality } \\
(9.4 \%)\end{array}$ & 2 & 0.19 & 0.06 & 0.67 \\
\hline \multicolumn{5}{|c|}{ Total } & 1,607 \\
\hline
\end{tabular}

\section{Conclusion}

In conclusion, we extracted 15 KPI from performance perspective and activity perspective for the TPM performance measurement of the company ' $\mathrm{D}$ '.

The 15 selected factors were divided into 4 perspectives according to the result of factor analysis for the BSC based activity performance measurement. We also used pair comparison analysis of AHP to calculate relative weight of each perspectives and the KPI of each standpoints and then measured economic value of each KPI based on the weights.

When corporations apply the study's methodology, selection of their own measure variables should be considered first to reflect its characteristics. We also suggest to refer to the indicated KPI selection used in this study. Overall, the corporation should be aware of the importance of performance measurement through conversion of qualitative value into economic value.

\section{References}

[1] C. Parker, "Performance Measurement," Work Study, Vol.49, No.2, pp.64-65, 2000.

[2] H. D. Moon, S. J. Song, Y. D. Jeong, K. S. Kang " Study on The Implementation and Application of POP
System Based on the Client/Server Architecture", Journal of Society of Korea Industrial and Systems Engineering, Vol 20, No. 42, pp.181-192, 1997.

[3] Hee J. Cho, "Study on the methodology for TPM Development and Performance Measurement", Korea University, Master dissertation, 2007.

[4] Hee. Y. Lee, "A Case Study on TPM Activities to Improve Overall Equipment Efficiency", Hanbat Univesity, Master Dissertation, 2009.

[5] K. L. Sedatole, "The Effect of Measurement Alternatives on a Nonfinancial Quality Measure's Forward: Looking Properties," The Accounting Review, Vol.78, 2003.

[6] Korean Agency for Technology and Standards Research on standards for terminology of equipment maintenance", pp.164-165. 2004.

[7] R. L. Lynch, K. F. Cross, Measure Up, Blackwell, pp.30-38, 1995.

[8] R. S. Kaplan, A. A. Atkinson, Advanced Management Accounting 3/E, Prentice Hall Inc, 1998.

[9] R. S. Kaplan, D. P. Norton, "The Balanced Scorecard-Measures that Drive Performance", Harvard Business Review. January, pp.71-79, 1992.

[10] Sang Y. Oh, "Verification of Quantifying BSC Qualitative Factors", Journal of Korea Academia-Industry Cooperation, Vol 8, No 2, pp.414-420, 2007.

[11] Sung Hyun Park, "Statistical Manufacturing Management", Minyonugsa, 1997.

[12] T. L. Saaty, The Analytic Hierarchy Process, McGraw Hill, 1980.

[13] T. S. Moon, H. J. Kim, S. B. Kang, "Implementation An EIP System for Mortor Component Industry", Korean Management Science Association/Korea Industry Engenneraing Association Spring Conference 2003회, pp.28-35, 2003.

[14] Young S. Lee, "Development of the key measurement indicators for the performance of TPM activity ", Aju University. Ph. D Dissertation, 2006.

[15] Yung S. Lee, Tae Y. Song, Hyo J Hahm, "Study on the TPM Performance Index by Applying KPI", Jounal Of The Korean Institute of Plant Engineering, Vol 10, No 3, pp.101 109, 2007. 
Sangyoung Oh

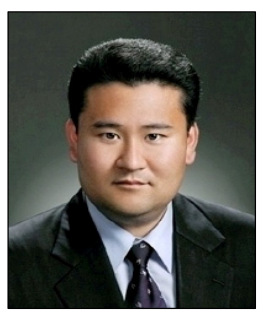

- Feb. 2001 : College of Business, Chungbuk Univ.(Ph. D.)

- Sept. $2010 \sim$ Current : Dept. of Business Administration, Youngdong Univ., Professor

$<$ Research Interests $>$

Knowledge Management, Innovation Theory, System Thinking, e-Biz, Government Policy

Yangkyun Son

[Regular Member]

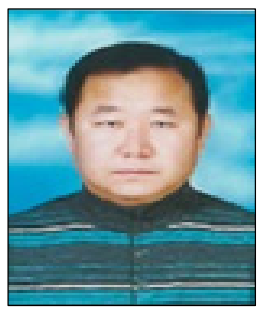

- Aug. 2011 : Graduate School of Yongdong Univ. (Master of Business Administration)

- Oct. $1989 \sim$ Current : DongSung Metal CO., LTD, Manager

$<$ Research Interests $>$

Customer Satisfaction, Marketing, Reliability and Maintenance Theory

\section{Yongsung Suh}

[Regular Member]

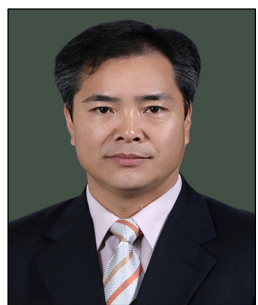

Feb. 1996 : College of Engineering, Sungkyunkwan Univ.(Ph. D.)

- Mar. $1996 \sim$ Current : Dept. of Business Administration, Youngdong Univ., Professor

$<$ Research Interests $>$

Quality Management, Operations Management, Reliability and Maintenance Theory

\section{Seonphil Jeong \\ [Regular Member]}

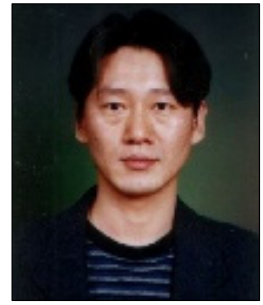

- Aug. 2007 : College of Business, Chungbuk Univ.(MIS. Ph. D.)

- Sept. $2008 \sim$ Current : Computer Science and Technology at BNU-HKBU United International College, Asst. Professor

$<$ Research Interests $>$

Knowledge Management Systems, System Analysis and Design 